\title{
Auto-Exposure Control Method for a Stereo Camera Robust to Brightness Variation
}

\author{
Hyun-Woo Kim, Soon Kwon, Jung Je-Kyo and JaeWook Ha \\ Division of IT-Convergence \\ Daegu Gyeongbuk Institute of Science and Technology, Daegu, Republic of Korea \\ Kimhw2109@dgist.ac.kr
}

\begin{abstract}
This paper proposes an AE (Auto-Exposure) control method for stereo carherós. Ymage processing has a problem that it shows different performance depending on brightness values even with the same image processing algorithm. Auto-exposure control solves this problem by maintaining the brightness value of the acquired image If preprocessing maintains the brightness value of the acquired image in a stereo canera, the performance of the imageprocessing algorithm becomes more stable. Traditional Auto-expostre control methods change the average brightness value of the image to attain the desired brightness value. However, this method is a disadvantageous in that it responds sensitively to exterior lighting sources and incurs low resource efficiency. The proposed method uses Gaussian sampling, with more sampling in the center region such that thesensitivity to light from exterior areas decreases and the resource efficiency improves. In experiments we confirmed that the performance of the proposed method is improved compared to that of existing methods.
\end{abstract}

Keywords: Auto-Exposure; Steked Camera, Gaussian Sampling; AE; AEC

\section{Introduction}

Recently, variors stucies using Kinect sensors, a product of Microsoft, have been conducted. Many people are becoming interested in research and companies related to stereo cameras.

The Kinect sensor is a device that uses infrared patterns. However, one disadvantage is that it works only indoors. Anstereo camera can utilize the disparity of observation spaces and the 3D shapes of objects throkgh its imaging capabilities. For this reason, stereo cameras are used in vehicles and robots, particularly because they are feasible in outdoor environments [1].

Cameras are sensitive to light, and image-processing algorithms are highly influenced by the brightness of the acquired image.

Traditiona methods respond sensitively to the light of exterior regions by means of brightness values of pixels for entire image regions. However, these methods are associated with unstable brightness information [2].

This paper proposes an auto-exposure control method for a stereo camera.

The proposed method a weight value using Gaussian sampling, which considers the stereo camera structure with an overlap region between different cameras.

The paper is organized as follows. Section 2 introduces the traditional auto-exposure method. Section 3 introduces the proposed method using Gaussian sampling considering the stereo camera structure. Section 4 compares the proposed method with the traditional method in experiments. Finally, Section 5 concludes the paper. 


\section{AE (Auto-Exposure) Control}

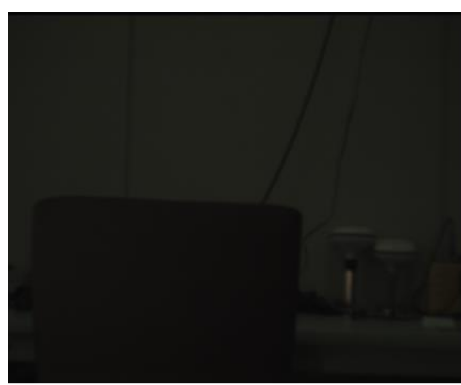

Underexposed

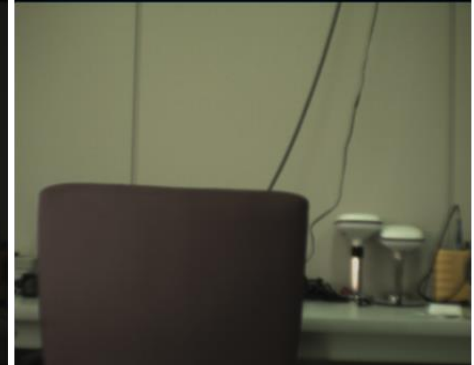

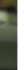

Overexposed

Figure 1. Brightness of images according to the exposure time

The brightness of images according to the exposure time is showh in Figure 1. Even when images are obtained in the same environment, the brightnes $s$ of the images depends on the exposure time. The brightness of an image increase with a longer exposure time and decreases with a shorter exposure time.

The performance of an image-processing algorithm such as one that processes detection and recognition steps is influenced by the brightness of the obtained images. Therefore, autoexposure control technology that maintains the brightnes 8 yalue of an acquired image is desirable [3].

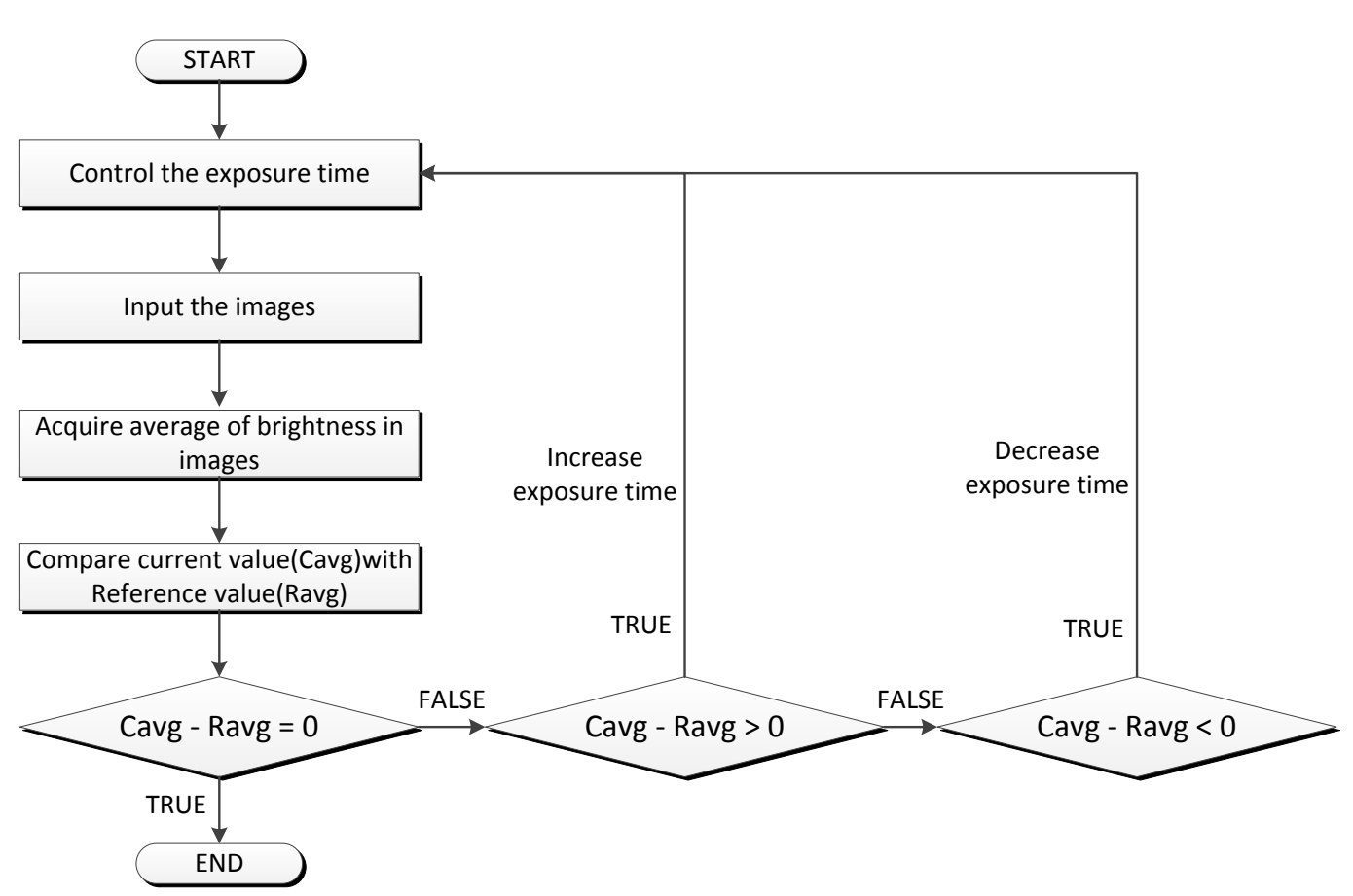

Figure 2. Auto-exposure control flow chart 
As shown in Figure 2, auto-exposure control computes the brightness of captured images and compares the brightness value with a reference value. It then controls the next exposure time. By decreasing the exposure time when the brightness value is higher than the reference value or by increasing the exposure time when the brightness value is lower than the reference value, the brightness of the acquired image is held constant [4-5].
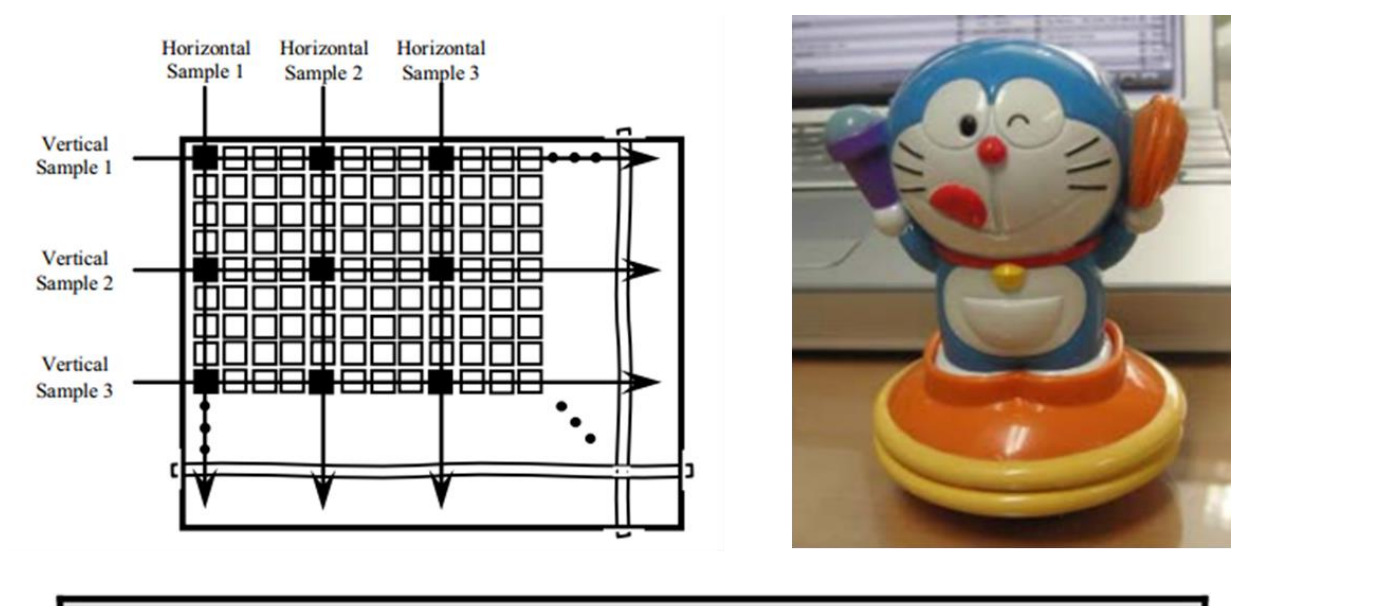

\begin{tabular}{|c|c|c|}
\hline \multicolumn{3}{|c|}{ Sample 1.299 x 221 Size Test Image } \\
\hline Application Pixels & Whole pixels & 1 16 sampling pixels \\
\hline Sum luminance & 7899242 & 508185 \\
\hline The number of pixel & 64090 & 4088 \\
\hline Average luminance & 123.25 & 124.31 \\
\hline
\end{tabular}

Figure 3, Pixel sampling method in another paper [6]

Figure 3 shows pixer sampling method in another paper. Usually average brightness of input image is computed by using the whole pixels of image. But in reference paper, average brightness is computed by using one pixel per 16 pixels for efficiency of little resource. As shown left infygure 3, it uses one pixel of the 4 pixels in row and one pixel of the 4 pixels in column to compute average brightness.

Table in Figure 3 shows the computation results comparing to the one using whole pixels of image. As shown in the table, there are no big difference between using the whole pixels of image and using 1/16 sampling pixels [6].

\section{Proposed Method}

The traditional auto-exposure control methods have problems when applied to a stereo camera. 


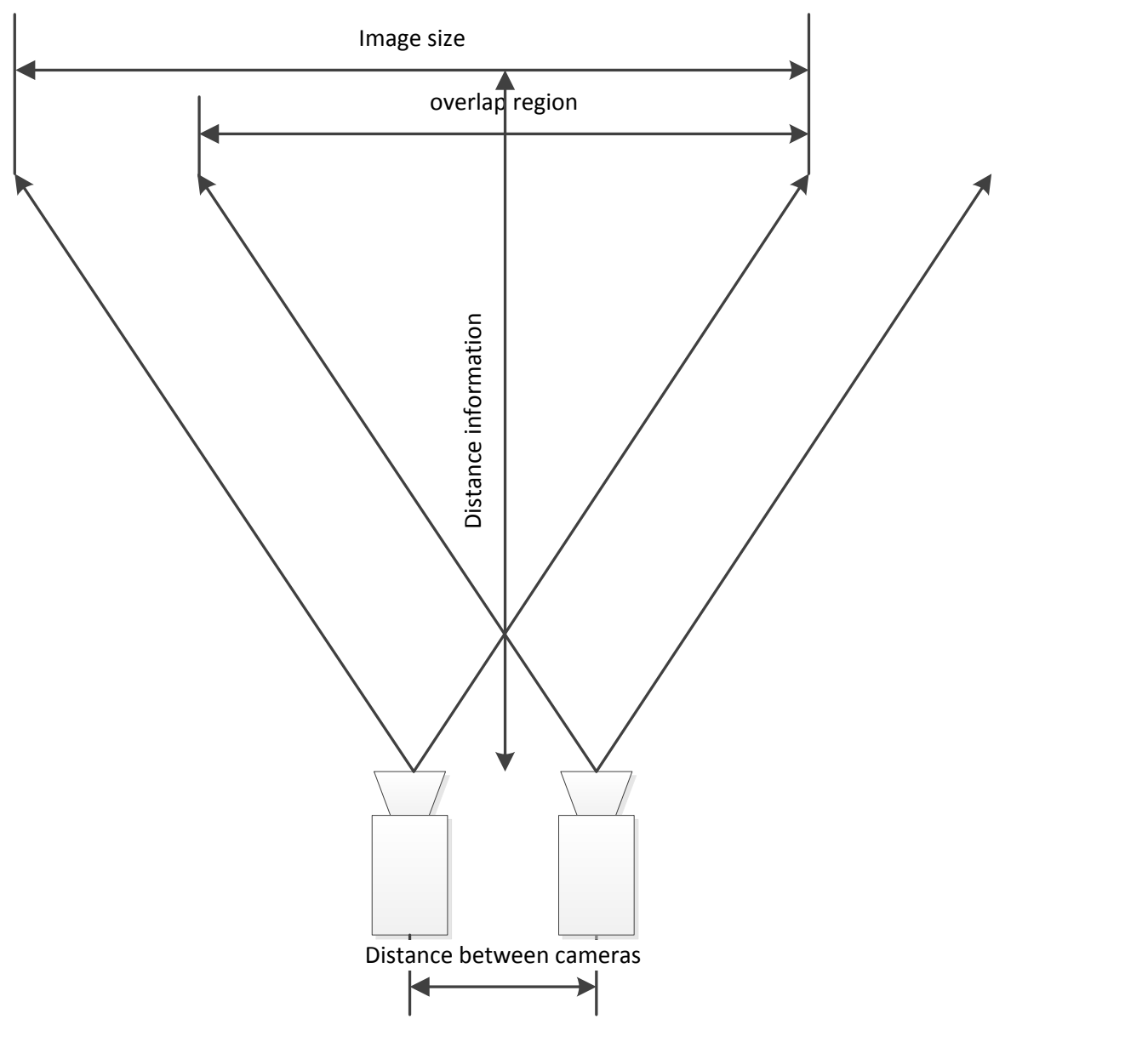

Figure 4. Stereo camera structure

A stereo camera pratching algorithn finds a disparity value in the overlapping region of the obtained left and right mages. However, as shown in Figure 4, a stereo camera has nonoverlapping regions depending on the distance between the two cameras. When applying the auto-exposure control method to the entire region in the images obtained by a stereo camera, instability arises due to the brightness values of non-overlapping regions [7].

To mitigate the problems that arise when using the entire image region, this paper proposes a Gaussian sampling method [8]. Generally, main objects or regions of interest are positioned in the center of an image.

As shown in Figure 5, with more sampling of the center region and less sampling of exterior regions, the method becomes less sensitive to the brightness of the exterior regions.

Additionally, considering the structure of a stereo camera moving the center point of the Gaussian pattern can be expressed by equation (1), which represents the shifting of the center point with the non-overlapping region of images on the left and the right. 


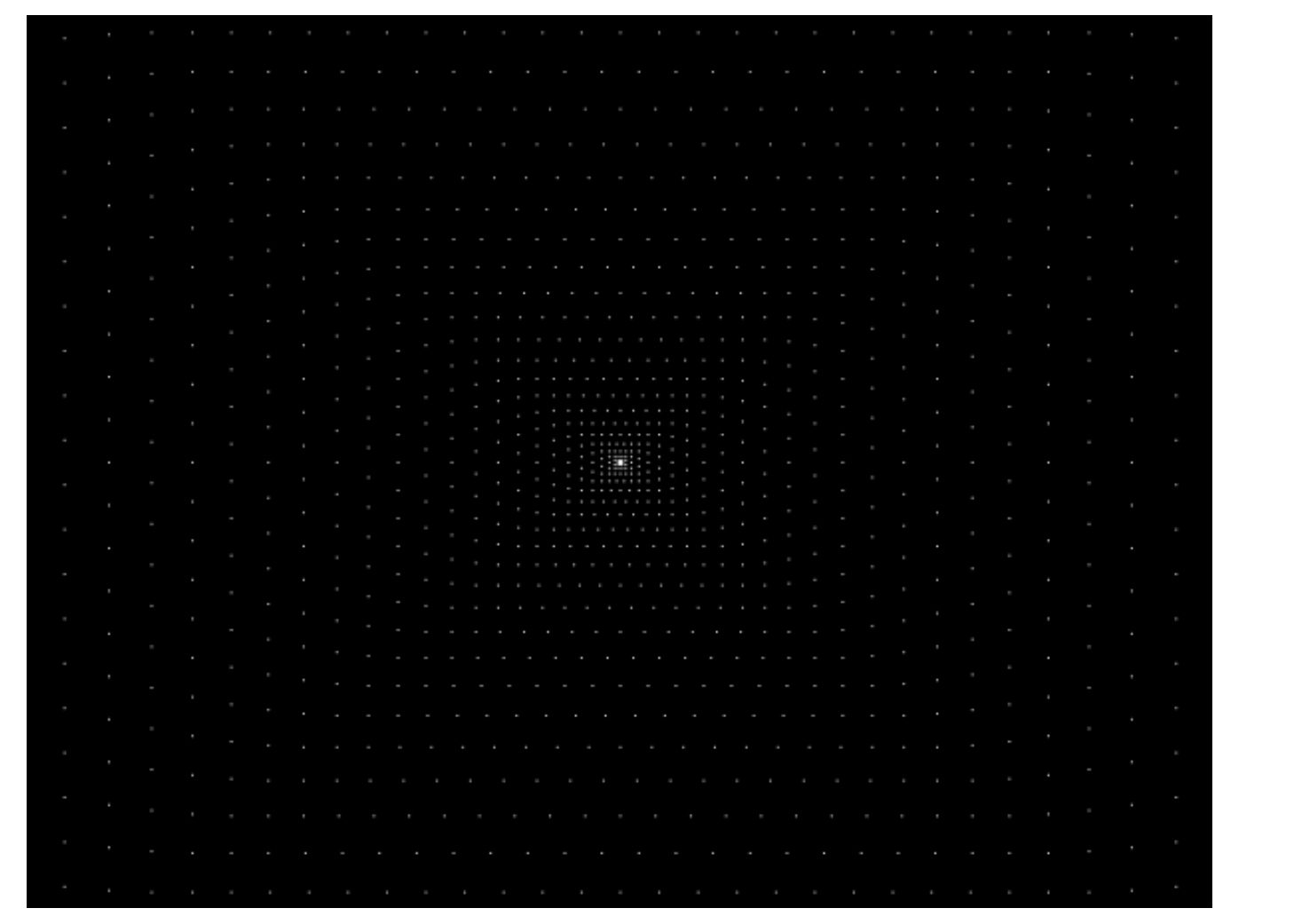

Figure 5. Gáussian sampling pattern

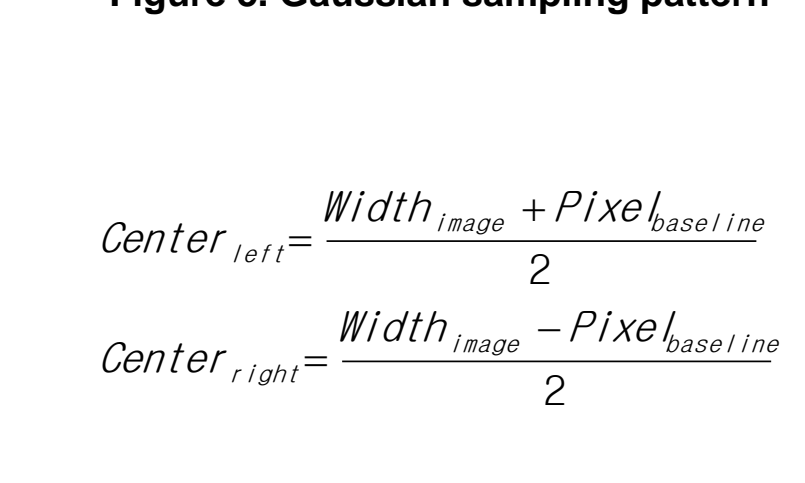

Figure 6 shows the result after shifting the center of the Gaussian pattern considering the overlapping region

The traditionalauto-exposure control methods have problems that they compute improper average brightness values for they are sensitive to the brightness of the exterior areas of images. In additions, they compute improper average brightness values using the brightness value of non-overlapping regions of the stereo camera. 


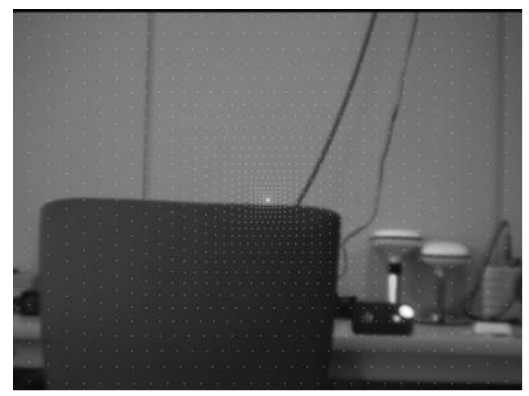

Left image

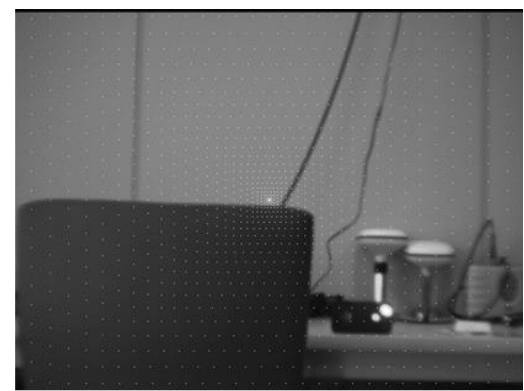

Right image

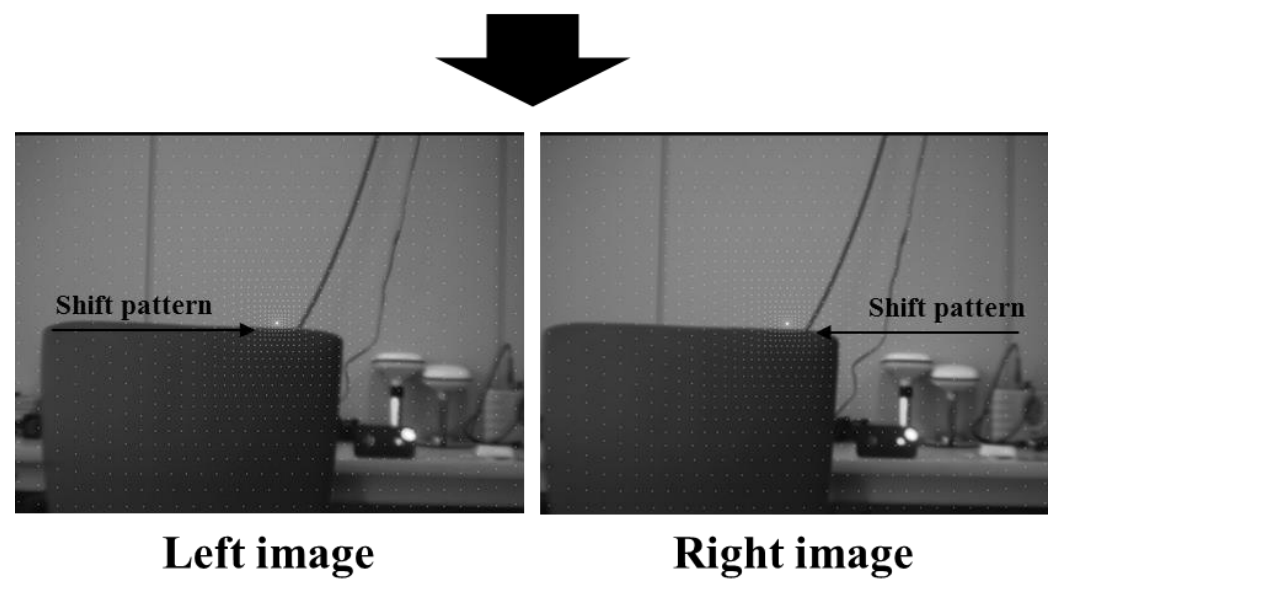

Figure 6. Result of Gaussian pattern shifting

\section{Experimental Results}

The paper compares the proposed Jmethod with existing methods to confirm the performance of the proposed method.

An experiment was done in anindoor condition that excluded light changes in order to compare the operating time and computed average brightness values of images.

Table 1 shows the compared results of the computed average brightness values. Comparing the proposed method with existing methods, the computed average brightness values are similar for the experiments done indoors, but the proposed method uses less pixel information.

Table 1. Average brightness comparison

\begin{tabular}{ccc}
\hline & $\mathrm{VGA}(640 * 480)$ & sized images (obtained by a stereo camera) \\
\hline Methods & Total pixels & Average brightness \\
\hline Whole pixels & 614,400 & 90 \\
\hline $1 / 16$ sampling pixels & 38,400 & 90 \\
\hline Proposed method & 2,308 & 92 \\
\hline
\end{tabular}




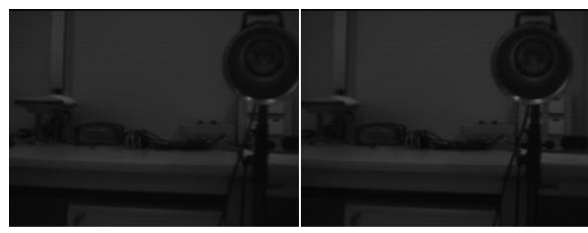

(a) Light off

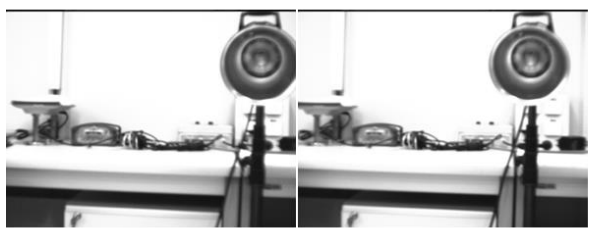

(c) Light off

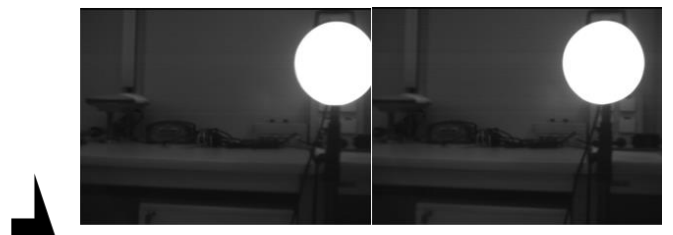

(b) Light on

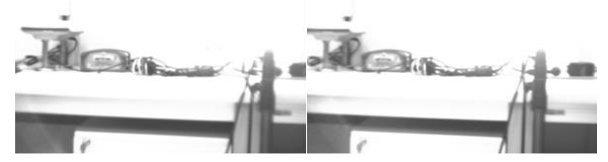

(d) Light on

Figure 7. Brightness variation due to exterior light

Figure 7 shows the input images used in the experiment to compare the brightness stability. The images have various brightness levels due to the light from the exterior area. In the figure, (a) and (b) are images obtained in a dark outdoor environment (c) and (d) are images obtained in a bright outdoor environment, and (b) and (d) are images captured with illuminating light in the exterior regions.

Figure 8 shows that the proposed Gaussian sampling method responds less sensitively than the whole pixels method according to a change in the exterior region. For (c) and (d), the brightness of the center region is high; therefore, the froposed method, which gives a higher weight to the center region, has a nigher average brightness value than the whole pixels method. However, the difference in the computed brightness values for images (c) and (d) is less when using the proposed method, indicating that it is less sensitive to changes in exterior regions than the whole pixels method.

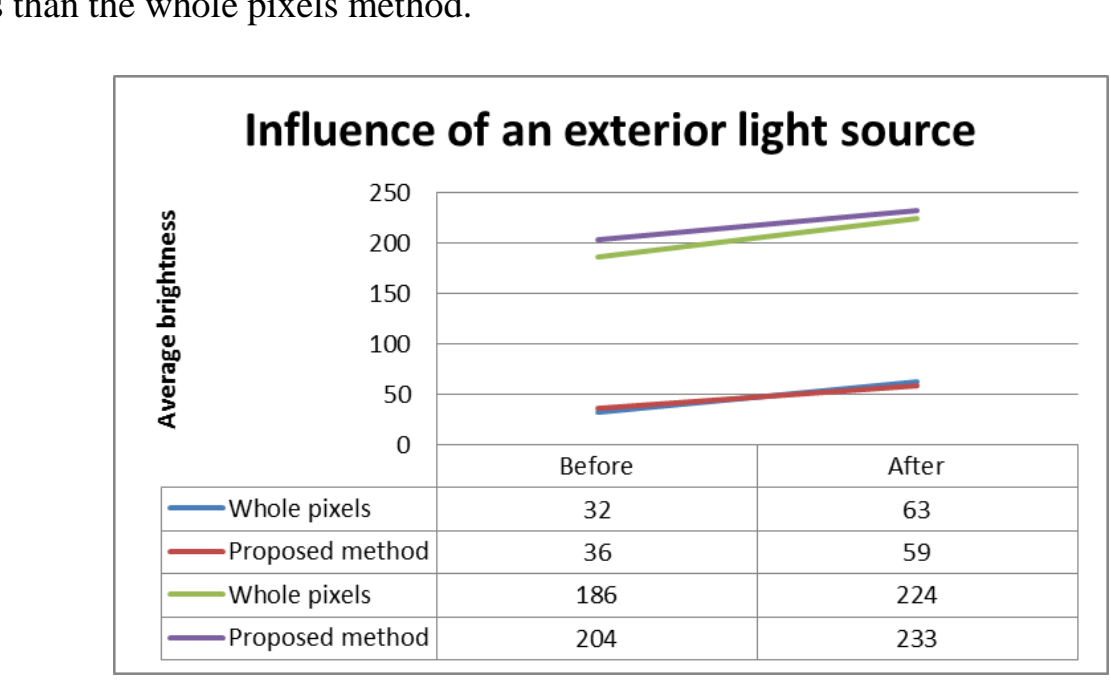

Figure 8. Influence of an exterior light source 


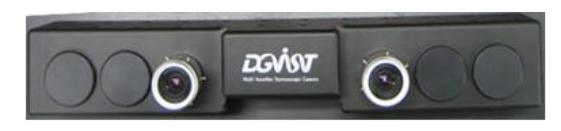

Stereo camera

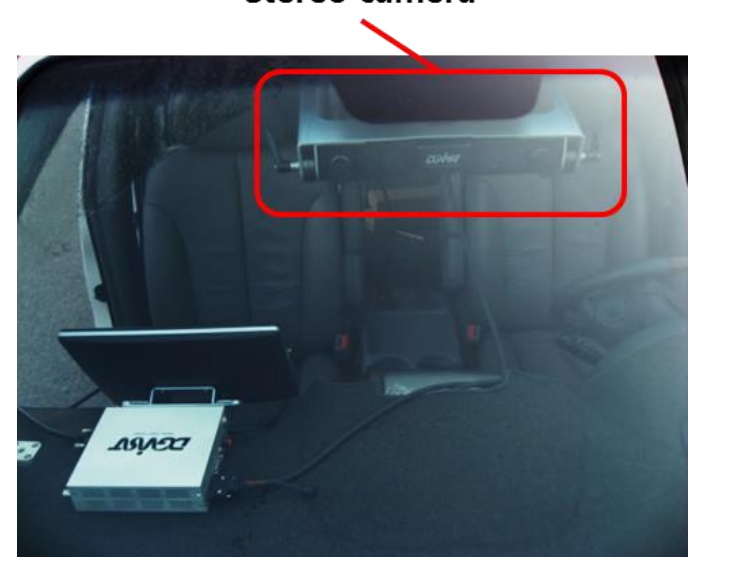

Figure 9. Stereo camera attached to a vehicle

A performance test was done using a stereo camera attached to a Nehicle, as shown in figure 9. Stereo camera is connected to the PC with Candink cable, and proposed method was applied with real-time processing. We obtained inages using the proposed $\mathrm{AE}$ and obtained images without using proposed $\mathrm{AE}$, then compared the results of stereo matching algorithm using them.

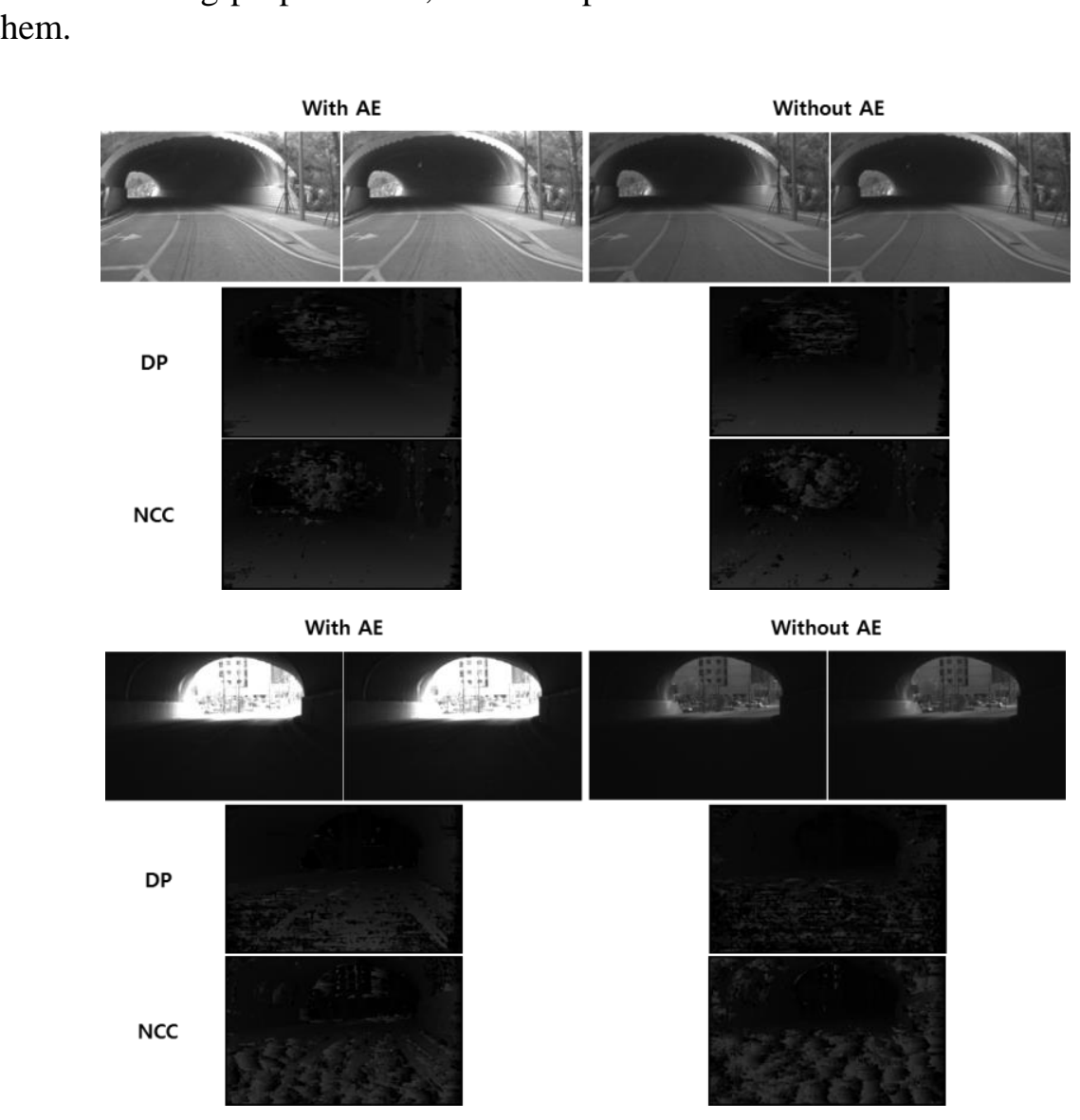




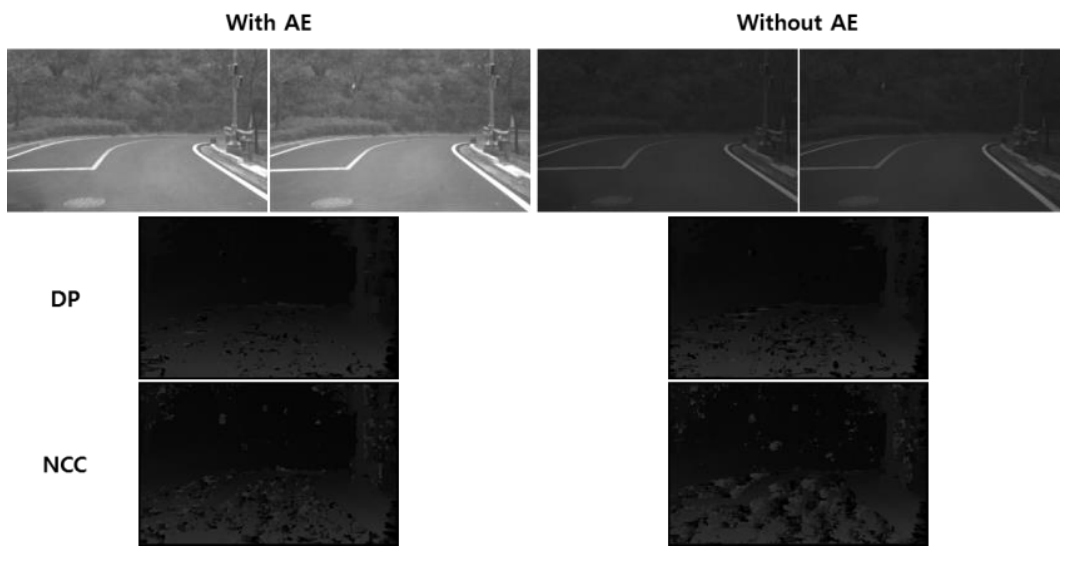

Figure 10. Performance comparison with the stereo-matching algorithms

Figure 10 shows the results when applying the proposed Ate metho in an outdoor environment and using DP(Dynamic Programming) and NCC(Normalized Cross Correlation) in stereo matching algorithm. The figure shows that the pesults of stereo matching have fewer holes when using the images with the proposed AE applied than using the images without the proposed AE method applied.

\section{Conclusions}

In this paper, a method to improve the peroormance of traditional AE method using Gaussian sampling is proposed. Comparing to the existing methods, the proposed method computes the brightness of images using fewer pixel images. Moreover, it is less sensitive to the brightness of exterior regions. It can also improve stereo matching algorithms in an outdoor environment. In this paper the AE method is applied by connecting a PC and stereo camera with a Camlink cable. For further work, the algorithm will be implemented in an ASIC(Application Specific Integrated Circuit) in a hardware design or will be implemented in an ISP(Image Sensor Processor) of a CIS(CMOS Image Sensor).

\section{Acknowledgements}

This work was supported by the DGIST R\&D Program of the Ministry of Education, Science and Technology of Korea (13-NB-0,5).

\section{References}

[1] N. Ahmed and I. N. Junejo, "A System for 3D Video Acquisition and Spatio-Temporally Conherent 3D Animation-Reconstruction using Multiple RGB-D Cameras", Proceedings of IJSIP, vol. 6, no. 2, (2013) April, pp. $113-128$.

[2] S. Shimizu, T. Kondo, T. Kohashi, M. Tsuruta and T. Komuro, "A new Algorithm for Exposure Control based on Fuzzy Logic for Video Cameras”, IEEE Trans. On Consumer Electronics, vol. 38, (1992) August, pp. 617-623.

[3] M. F. Al-Samaraie, "A new Enhancement Approach for Enhancing Image of Digital Cameras by Changing the Contrast", Proceedings of IJAST, vol. 32, (2011) July, pp. 13-22.

[4] J. S. Lee, Y. Y. Jung, B. S. Kim and S. J. Ko, "An Advance Video Camera System with Robust AF, AE, and AWB Control”, IEEE Trans. On Consumer Electronics, vol. 47, no. 3, (2001) August, pp. 694-699.

[5] T. Kuno, H. Sugiura and N. Matoba, "A New Automatic Exposure System for Digital Still Cameras", IEEE Trans. On Consumer Electonics, vol. 44, no. 1, (1998) February, pp. 192-199. 
[6] K. G. Kim, J. Y. Ha and B. S. Kang, "The Algorithm and Hardware Implementation of Average Luminance Computation Method of Image to Improve Auto Exposure in Mobile Camera”, Proceedings of ICEIC, (2008) June, pp. 24-27.

[7] A. Agarwal, "Compressing Stereo Images Using a Reference Image and the Exhaustive Block Matching Algorithm to Estimate Disparity between the Two Images", Proceedings of IJAST, vol. 32, (2011) July, pp. $1-12$.

[8] J. Y. Liang, Y. J. Qin and Z. L. Hong, “An Auto-exposure Algorithm for Detecting High Contrast Lighting Conditions”, IEEE International Conference on ASICON, (2007) October, pp. 725-728.

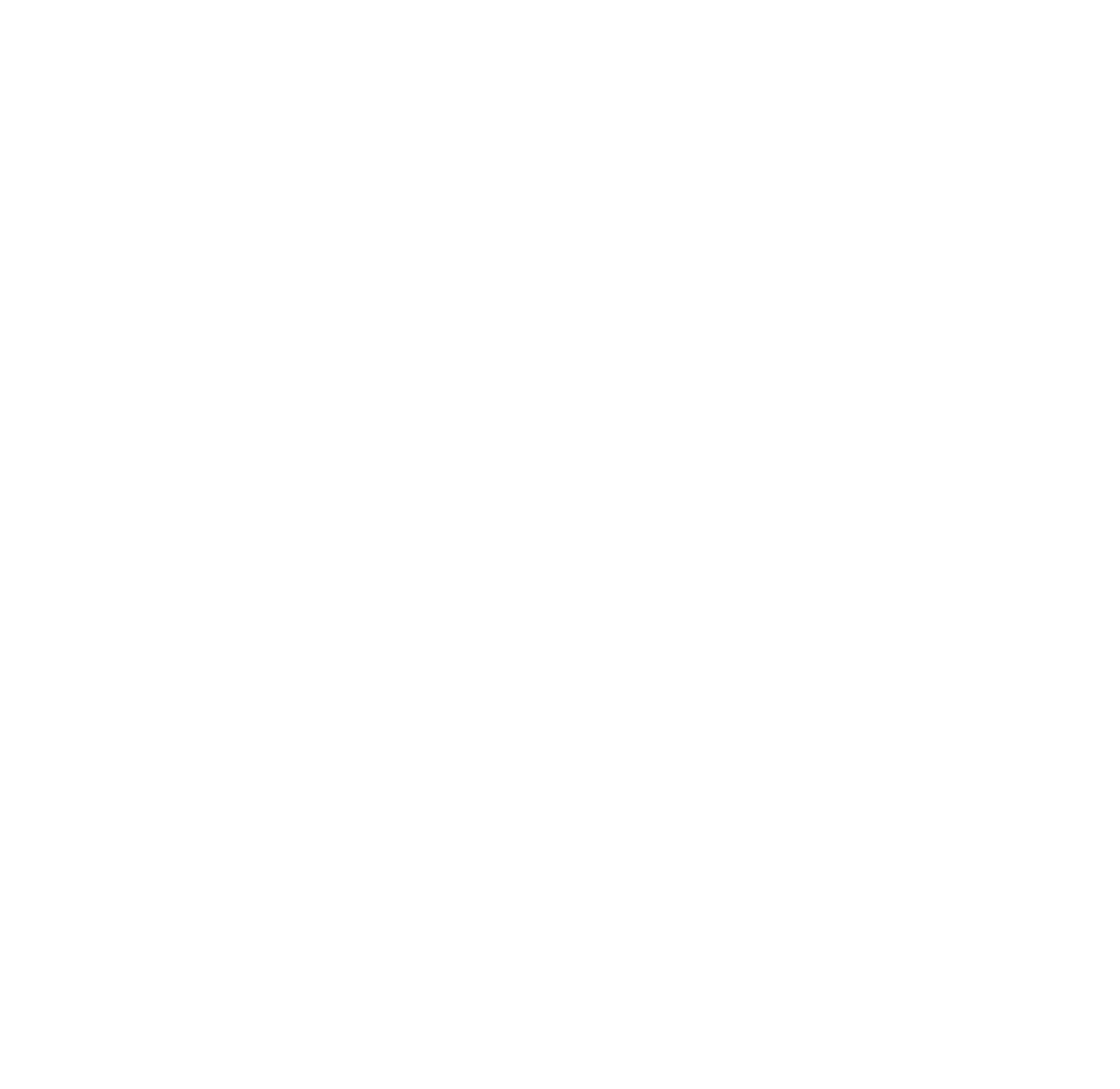

